\title{
Amount of antiprotons in cosmic rays due to halo neutralino annihilation
}

\author{
A. Bottino, C. Favero, N. Fornengo, G. Mignola \\ Dipartimento di Fisica Teorica, Università di Torino and INFN, Sezione di Torino, via P. Giuria 1, 10125 Torino, Italy ${ }^{1}$
}

Received 13 July 1994

\begin{abstract}
We evaluate the antiproton-to-proton flux ratio which can be generated by neutralino-neutralino annihilation in the galactic halo, considering the most general compositions for the relic neutralinos and modelling the neutralino local density according to its relic abundance. We find that in the case of mixed neutralino compositions this $\bar{p} / p$ ratio is higher than for pure higgsinos or gauginos. It is shown how the expected improvements in sensitivity for the new measurements of the $\bar{p} / p$ may provide very useful information, complementary to the one obtainable with other experimental means.
\end{abstract}

\section{Introduction}

The neutralino $(\chi)$ is generally considered the most favorite candidate for cold dark matter, and many possible ways for finding some evidence for it as a relic particle in the Universe have been envisaged. The most straightforward mean is obviously provided by the direct method, where the incoming of a dark matter (DM) neutralino would be signalled by the energy released, in an appropriate detector, by this particle when it scatters off a nucleus of the experimental apparatus $[1,2]$. The existence of relic neutralinos could also be inferred indirectly, by detecting some specific signals due to neutralino-neutralino annihilations. The best chances of obtaining indirect information of this sort would be provided by neutrino telescopes aiming at the measurement of fluxes of high-energy neutrinos from the Sun or from the centre of the Earth [3,4]. In fact these macroscopic bodies could efficiently cap-

\footnotetext{
${ }^{1}$ E-mail addresses:

(bottino, c_favero, fornengo, mignola) @to.infn.it
}

ture and accumulate relic neutralinos, and finally emit the neutrinos produced by the neutralino pair annihilation in their interior. To be sensitive to this measurement the neutrino telescopes have to be of very large area $\left(>10^{5} \mathrm{~m}^{2}\right)[4]$. Other indirect methods consist in the detection of signals due to $\chi-\chi$ annihilation occurring in our galactic halo [5]. Theoretical predictions for the magnitude of these signals have been performed in the case of the gamma-line [6-8], of a diffuse gamma-ray flux [7-9], of a gamma-ray flux from the center of the Galaxy $[10,8]$ and of $\bar{p}, e^{+}$ components in the cosmic rays [11-14].

The issue at stake, i.e. the detection of a DM particle in our Universe, is so important that every method of detection is worth to be pursued. This is even more true, since the odds of the various experimental means depend on the actual nature (composition and mass) of the neutralino. In the present paper we concentrate on the evaluation of the amount of antiprotons in cosmic rays due to the $\chi-\chi$ annihilation in our halo. This problem has been previously addressed by other authors only for neutralinos of pure compositions: ei- 
ther higgsinos or gauginos, and for assigned values of the neutralino relic abundance [11-14]. At variance with these previous investigations we consider here neutralinos of the most general compositions and we model the neutralino local density according to its calculated relic abundance. Our results show how sensitive may be the antiproton-to-proton flux ratio, $\bar{p} / p$, to the nature of the neutralino. We find that the experimental capabilities are not far from the possibility of a fruitful search for neutralino dark matter by measuring the $\bar{p} / p$ ratio. The chances of obtaining positive results in these experiments depend on the actual nature of the neutralino, these chances being higher for a $\chi$ of mixed composition than for the exactly pure configurations.

\section{The $\bar{p}$ flux}

The differential rate (per unit volume) for production of $\bar{p}$ 's from $\chi-\chi$ annihilation is given by

$$
\frac{d S}{d E_{\bar{p}}}=\langle\sigma v\rangle f\left(E_{\bar{p}}\right)\left(\frac{\bar{\rho}_{\chi}}{m_{\chi}}\right)^{2}
$$

where $E_{\bar{p}}$ is the $\bar{p}$ energy, $\sigma$ is the $\chi-\chi$ annihilation cross section, $v$ the neutralino velocity in the halo, and $\bar{\rho}_{\chi}$ is an averaged neutralino density to be discussed later; $f\left(E_{\bar{p}}\right)$ denotes the $\bar{p}$ differential spectrum

$$
\begin{gathered}
f\left(E_{\bar{p}}\right) \equiv \frac{1}{\sigma} \frac{d \sigma(\chi \chi \rightarrow \bar{p}+X)}{d E_{\bar{p}}} \\
=\sum_{F, f} B_{\chi f}^{(F)} \frac{d N_{\bar{p}}^{f}}{d E_{\bar{p}}}
\end{gathered}
$$

where $F$ denotes the $\chi-\chi$ annihilation final states, $B_{\chi f}^{(F)}$ is the branching ratio into quarks or gluons in the channel $F ; d N_{\tilde{p}}^{f} / d E_{\bar{p}}$ is the differential distribution of the antiprotons generated by hadronization of quarks (with the exception of the top quark) and of gluons.

From Eq. (1) the standard formula for the interstellar $\bar{p}$ flux, due to $\chi-\chi$ annihilation, follows

$$
\Phi_{\bar{p}}\left(E_{\bar{p}}\right)=\frac{1}{4 \pi}\langle\sigma v\rangle f\left(E_{\bar{p}}\right)\left(\frac{\bar{\rho}_{X}}{m_{\chi}}\right)^{2} v_{\bar{p}} \tau_{\bar{p}}
$$

where $v_{\bar{p}}$ and $\tau_{\bar{p}}$ are the $\bar{p}$ velocity and confinement time, respectively.
Of the various quantities that enter in Eq. (3) the first two, $\langle\sigma v\rangle$ and $f\left(E_{\bar{p}}\right)$, depend on the neutralino properties, whereas the last two, $v_{\bar{p}}$ and $\tau_{\bar{p}}$, are only related to the propagation of $\bar{p}$ 's inside the galactic halo. The quantity $\bar{\rho}_{X}$ depends on the intrinsic properties of $\chi$, but also on the features of the $\bar{p}$ propagation, since the average has to be taken over the region where the $\bar{p}$ 's diffuse. In what follows we illustrate how we evaluated all these quantities.

\subsection{The $\chi-\chi$ annihilation cross section}

In the previous works [11-14] $\langle\sigma v\rangle$ has been estimated by assigning an arbitrary value to the neutralino relic abundance $\Omega_{X} h^{2}$ and by exploiting a standard expression relating the relic abundance to the $\chi-\chi$ annihilation cross section integrated from the freeze-out temperature to the present temperature [15]. Instead, we calculated $\langle\sigma v\rangle$ in the framework of a particle model, the Minimal Supersymmetric Standard Model (MSSM). For the evaluation of $\sigma$ we have included all the relevant diagrams at the tree-level, i.e. Higgsexchange diagrams and Z-exchange diagrams in the s-channel and diagrams with exchanges of squarks, neutralinos and charginos in the t-channel, with all the final states which may contribute to the production of $\bar{p}$ 's. We have also included the one-loop diagrams which generate a two-gluon final state [16], since, as shown in Ref.[14], this contribution is important for $\bar{p}$ production by $\chi^{-} \chi$ annihilation in case of neutralinos with gaugino-dominance and with a mass $m_{\chi}$ lighter than the top mass.

Our calculations have been performed for neutralinos of the most general composition in terms of the photino, zino and higgsino fields

$$
\chi=a_{1} \tilde{\gamma}+a_{2} \tilde{Z}+a_{3} \tilde{H}_{1}^{0}+a_{4} \tilde{H}_{2}^{0}
$$

$\tilde{\gamma}$ and $\tilde{Z}$ are related to the $U(1)$ and $S U(2)$ neutral gauginos, $\tilde{B}$ and $\tilde{W}_{3}$, by the standard rotation in terms of the Weinberg angle. For other details about the theoretical framework adopted here for our calculations we refer to our previous paper [17]. We only remind that the neutralino sector is usually described in terms of three parameters: $M_{2}, \mu$ and $\tan \beta$. In the present paper for $\tan \beta$ we consider two representative values $\tan \beta=2,8$ whereas the other two parameters are var- 
Table 1

Valucs for the $p_{i}$ 's parameters of the distribution given in Eq. (5) for the value $E_{\mathrm{cm}} / 2=30 \mathrm{GeV}$. Each row corresponds to a quark flavour and to the gluon.

\begin{tabular}{lllll}
\hline & $p_{1}$ & $p_{2}$ & $p_{3}$ & $p_{4}$ \\
\hline$u, d$ & 1.7443 & 2.9722 & -0.62737 & -1.1193 \\
$s$ & 7.5915 & 4.0079 & 1.5257 & -1.4500 \\
$c$ & 36.268 & 4.8055 & 3.0725 & -1.5923 \\
$b$ & 6.9350 & 5.4031 & 1.7076 & -1.3286 \\
gluon & 132.86 & 4.9732 & 3.5982 & -1.6360 \\
\hline
\end{tabular}

ied in the ranges: $10 \mathrm{GeV} \leq M_{2} \leq 6 \mathrm{TeV}, 10 \mathrm{GeV}$ $\leq|\mu| \leq 3 \mathrm{TeV}$.

We introduce a composition parameter $P=a_{1}{ }^{2}+$ $a_{2}{ }^{2}$ which gives the gaugino fractional weight. Almost every where in the parameter space, at fixed $\tan \beta$, there is a one-to-one correspondence: $\left(M_{2}, \mu\right) \leftrightarrow\left(P, m_{\chi}\right)$; thus in what follows we replace the two parameters $\left(M_{2}, \mu\right)$ with $\left(P, m_{\chi}\right)$, since these variables have a more direct physical meaning for the neutralino.

As for the unknown masses (i.e. masses for Higgs bosons, Susy particles and top quark), it has to be specified that most of the results of our analysis presented here refer to the following set of values: $m_{t}=$ $170 \mathrm{GeV}, m_{h}=50 \mathrm{GeV}, m_{\tilde{f}}=1.2 m_{X}$, when $m_{X}>45$ $\mathrm{GeV}, m_{\tilde{f}}=45 \mathrm{GeV}$ otherwise, except for the mass of the top scalar partners (the only ones relevant to radiative corrections) which has been taken $\tilde{m}=1 \mathrm{TeV}$. Only in a few cases, other choices, to be defined later, have been adopted for some of these masses.

\subsection{The $\bar{p}$ differential spectrum}

Let us consider now the $\bar{p}$ differential distribution $f\left(E_{\bar{p}}\right)$. The branching ratios $B_{\chi f}^{(F)}$ have been calculated for all annihilation final states which may produce $\bar{p}$ 's. These states fall into two categories: i) direct production of quarks and gluons, ii) generation of quarks through intermediate production of Higgs bosons, gauge bosons and $t$ quark. In order to obtain the distributions $d N_{\bar{p}}^{f} / d E_{\bar{p}}$ the hadronization of quarks (with the exception of the top quark) and gluons has been evaluated by using the Monte Carlo code Jetset $7.2[18,19]$.

The ensuing differential distributions show the usual scaling properties in the variable $x=E_{\bar{p}} /\left(E_{\mathrm{cm}} / 2\right)$, where $E_{\mathrm{cm}}$ is the center of mass energy $[20,13]$. However, sizeable violations of this scaling behavior occur

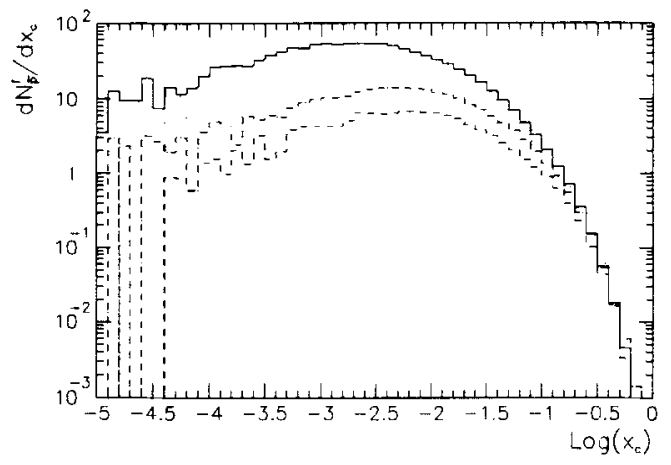

Fig. 1. $\bar{p}$ differential distibution $d N_{\bar{p}}^{f} / d x_{c}$ from the fragmentation of a $b$ quark, as a function of $x_{c}=T_{\bar{p}} /\left(E_{\mathrm{cm}} / 2\right), T_{\bar{p}}$ being the antiproton kinetic energy. The curves refer to four different values of $E_{\mathrm{cm}}$. From top to bottom: $E_{\mathrm{cm}} / 2=120,60,30$ and $15 \mathrm{GeV}$.

for small values of $x(x \lesssim 0.1)$ [20]. Since this is precisely the energy range of interest for the process under discussion, instead of using these scaling properties, we have evaluated the differential distributions at four different values: $E_{\mathrm{cm}} / 2=15,30,60,120 \mathrm{GeV}$. The differential distributions at different $E_{\mathrm{cm}}$, have been obtained by interpolation. A convenient analytic expression that fits our numerical spectra is provided by the following form

$$
\frac{d N_{\bar{p}}^{f}}{d x}=p_{1}|w|^{p_{2}} \exp -\frac{1}{2}\left(\frac{w-p_{3}}{p_{4}}\right)^{2}
$$

where $w=\log \left(x_{c}\right), x_{c}=T_{\bar{p}} /\left(E_{\mathrm{cm}} / 2\right)$ and $T_{\bar{p}}$ is the $\bar{p}$ kinetic energy. The parameters $p_{i}$ 's are given in Table 1 for the value $\left(E_{\mathrm{cm}} / 2\right)=30 \mathrm{GeV}$.

As an example, our results for the case of the differential distribution due to the $b$ quark fragmentation are shown in Fig. 1 in terms of the variable $x_{c}$. Our spectra are numerically in agreement with the ones of Ref. [13] for the cases considered in that paper, i.e. 
for the fragmentations of the $c$ and $b$ quarks.

\subsection{Propagation of the $\bar{p}$ 's in the halo}

Let us turn now to the evaluation of the quantities that depend on properties of the $\bar{p}$ propagation in the halo. In the diffusion model [21] for propagation of cosmic rays in our Galaxy the confinement time $\tau_{\bar{p}}$ may be evaluated from the formula

$$
\begin{aligned}
\tau_{\bar{p}} & =\frac{h_{h}^{2}}{3 D} \frac{1-\frac{1}{2}\left(h_{g} / h_{h}\right)^{2}+\frac{1}{8}\left(h_{g} / h_{h}\right)^{3}}{1-\frac{1}{2}\left(h_{g} / h_{h}\right)} \\
& \simeq \frac{h_{h}^{2}}{3 D}
\end{aligned}
$$

where $h_{g}$ and $h_{h}$ are the half-thicknesses of the disk and of the confinement region, respectively, and $D$ is the diffusion coefficient. The approximation involved in Eq. (6) is justified since $h_{g}=100-150 \mathrm{pc}$ and $h_{h} \gtrsim$ $700 \mathrm{pc}$. By varying $h_{h}$ over the range $1 \mathrm{Kpc} \leq h_{h} \leq$ $10 \mathrm{Kpc}$ and by using for $D$ the numerical values of Ref. [21] (the ratio $\mathrm{D} / h_{h}$ is rather stable with values in the range $\left.D / h_{h} \simeq(1.7-3.3) \cdot 10^{6} \mathrm{~cm} \mathrm{~s}^{-1}\right)$, one obtains for the $\bar{p}$ confinement time

$$
0.3 \cdot 10^{15} \mathrm{~s} \lesssim \tau_{\bar{p}} \lesssim 6 \cdot 10^{15} \mathrm{~s}
$$

in agreement with previous evaluations $[12,13]$. In the following, for definiteness, we adopt the value $\tau_{\bar{p}}=$ $3 \cdot 10^{15} \mathrm{~s}$.

Let us discuss now the quantity $\bar{\rho}_{\chi}$ appearing in Eq. (3). This represents the average value of the neutralino density over a sphere centered at the Earth position, with a radius of the order of $h_{h}$. It is then straightforward to relate $\bar{\rho}_{\chi}$ to the local (solar neighborhood) neutralino density $\rho_{X}$, loc . Assuming for the DM halo the usual distribution $\rho(r)=\rho_{\text {loc }}\left(1+r_{\odot}^{2} / a^{2}\right) /(1+$ $\left.r^{2} / a^{2}\right)$, where $r_{\odot} \simeq 8.5 \mathrm{kpc}$ and $\rho_{\mathrm{loc}} \simeq 0.3 \mathrm{GeV} \mathrm{cm}^{-3}$ one finds in general

$$
\begin{aligned}
& \left(\left\langle\rho^{2}\right\rangle / \rho_{\mathrm{loc}}^{2}\right)^{1 / 2} \simeq 1.8 \\
& \text { for } a=2.0 \mathrm{kpc}[22], \\
& \left(\left\langle\rho^{2}\right\rangle / \rho_{\mathrm{loc}}^{2}\right)^{1 / 2} \simeq 0.97 \\
& \text { for } a=7.8 \mathrm{kpc}[23] .
\end{aligned}
$$

Here, to be conservative, we adopt an estimate close to the one in Eq. (9), $\bar{\rho} \simeq \rho_{\mathrm{loc}}$, which entails a similar relationship for the fraction of DM due to neutralinos, i.e. $\bar{\rho}_{\chi} \simeq \rho_{\chi \text {,loc }}$.

\subsection{Evaluation of $\rho_{\chi}$,loc}

In order to assign a value to $\rho_{\chi}$,loc, for any point of the parameter space (specified by a set of values for $M_{2}, \mu$ and $\tan \beta$ ), we have evaluated the neutralino relic abundance $\Omega_{\chi} h^{2}$ and we have then determined the value $R \equiv \min \left\{1,\left(\Omega_{\chi} h^{2}\right) /\left(\Omega h^{2}\right)_{\min }\right\}$, where $\left(\Omega h^{2}\right)_{\min }$ is defined as the minimum value of $\left(\Omega h^{2}\right)$ compatible with observations. Typically, taking $\Omega \gtrsim 0.1$ and $h \simeq 0.5$, one has $\left(\Omega h^{2}\right)_{\min } \simeq 0.03$; this is in fact the value employed here for $\left(\Omega h^{2}\right)_{\min }$. Then $\rho_{\chi, \text { loc }}$ has been determined by the standard rescaling procedure

$$
\rho_{\chi, \mathrm{loc}}=R \rho_{\mathrm{loc}} .
$$

The method adopted for the evaluation of $\Omega_{\chi} h^{2}$ is the one illustrated in Ref. [17]. We only remind here that $\Omega_{X} h^{2}$ is inversely proportional to the averaged annihilation cross section $\langle\sigma v\rangle$, integrated from the freeze-out temperature to the present one. Thus it follows that when, in a given point of the parameter space, $\sigma$ is large, in that point $\Omega_{X} h^{2}$ is depleted.

\subsection{Modulation of the $\bar{p}$ flux}

The $\bar{p}$ flux, measured at Earth, may be obtained from the interstellar $\bar{p}$ flux given by Eq. (3) by taking into account the modulation due to the solar wind. This effect is calculated here by employing the procedure discussed in Refs. [24,25] and also utilized in Refs. [11-14]. Denoting by $E$ and $T$ the $\bar{p}$ total and kinetic energies and by $k$ the $\bar{p}$ momentum measurable at Earth, the solar-modulated $\bar{p}$ flux is given by

$$
\Phi_{\bar{p}}(E)=\frac{E^{2}-m_{p}^{2}}{E_{\bar{p}}^{2}-m_{p}^{2}} \Phi_{\bar{p}}\left(E_{\bar{p}}\right) .
$$

$E$ and $E_{\bar{p}}$ are related by

$$
\begin{aligned}
& E_{\bar{p}}=k_{c} \ln \frac{k+E}{k_{c}+E_{c}}+E_{c}+\Delta E \\
& \quad \text { for } k<k_{c}, \\
& E_{\bar{p}}=E+\Delta E \quad \text { for } k \geq k_{c} .
\end{aligned}
$$

The numerical values for $k_{c}$ and $\Delta E$ depend on the phase of the 11 year solar cycle. In what follows only the antiproton-to-proton flux ratio $\bar{p} / p$, rather than the individual $\bar{p}$ and $p$ fluxes, will be considered. Since 


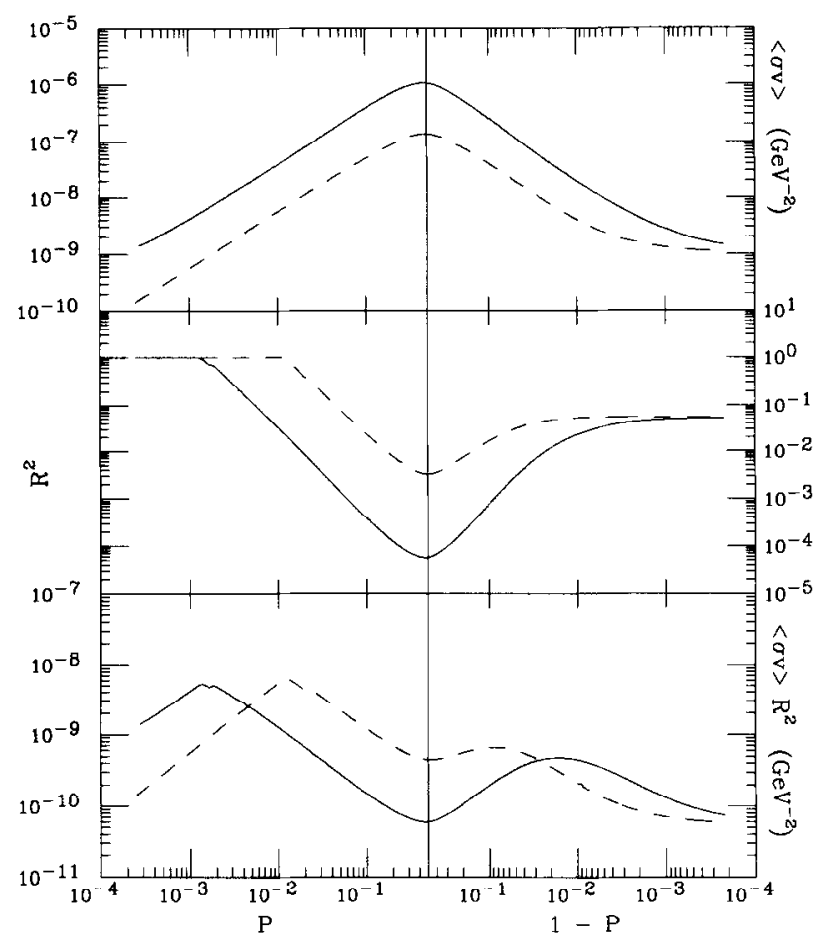

Fig. 2. $\langle\sigma v\rangle, R^{2}$ and $\langle\sigma v\rangle R^{2}$ as functions of the neutralino composition at a fixed value of the neutralino mass $m_{X}=30 \mathrm{GeV}$. The other parameters are: $\tan \beta=8$ (solid curve), $\tan \beta=2$ (dashed curve), $m_{h}=50 \mathrm{GeV}, m_{f}=45 \mathrm{GeV}$. In these plots, in order to represent the dependence of various quantities accurately for very small mixings (i.e. for $P \sim 0, P \sim 1$ ), on the left part of the horizontal axis the variable $P$ is given, whereas on the right part of the same axis the complementary variable $1-P$ is reported, both in a log-scale.

these two fluxes are modulated in the same way, $\bar{p} / p$ is quite insensitive to the specific values adopted for $k_{c}$ and $\Delta E$. To be definite we use in the following the values corresponding to a period of minimum solar activity: $k_{c}=1.015 \mathrm{GeV}$ and $\Delta E=495 \mathrm{MeV}$.

\section{Results and conclusions}

From Eqs. (3) and (10) we see that, at fixed $m_{\chi}$, the flux $\Phi_{\bar{p}}$ depends on the neutralino properties mainly through the quantity $\langle\sigma v\rangle R^{2}$ (some further, less effective dependence, is introduced in $\Phi_{\bar{p}}$ through $f\left(E_{\bar{p}}\right)$ ). Thus we start the presentation of our results by discussing the behavior of $\langle\sigma v\rangle R^{2}$ as we move in the neutralino parameter space. In Fig. 2 we display $\langle\sigma v\rangle, R^{2}$ and their product $\langle\sigma v\rangle R^{2}$ as functions of $P$, for $m_{\chi}=$ $30 \mathrm{GeV}, \tan \beta=2,8$ and for the set of mass parameters ( $m_{h}$ and $m_{\tilde{f}}$ ) previously stated. In this figurc we note a number of interesting properties: i) the $\chi-\chi$ annihilation cross section is maximal for large higgsino- gaugino mixing ( $P \simeq 0.5$ ) (this feature is expected, since the small value used here for $m_{h}$ makes the Higgs-exchange amplitude of the annihilation cross section very large and dominant over the other amplitudes; this effect is particularly important when $\chi$ is largely mixed); ii) the neutralino relic abundance $\Omega_{\chi} h^{2}$, and then also $R^{2}$, are strongly depleted except for rather pure compositions, since the annihilation cross section is very efficient unless $P \simeq 0$ or $P \simeq 1$ (for the reason mentioned at point i)); iii) from the plot of $\langle\sigma v\rangle R^{2}$ one sees that the combination of the two previous properties makes the neutralinos with a mixed composition ( $P \simeq 10^{-3}-10^{-2}$ in the present representative points) as the most favourite ones for detection by the method under discussion; iv) exactly pure compositions $(P=0,1)$ provide rather low values for $\langle\sigma v\rangle R^{2}$ and are in general not indicative of what the real situation could be when some mixing is turned on.

In Fig. 3 we show how the quantity $\langle\sigma v\rangle R^{2}$ depends on the values assigned to the masses $m_{h}$ and $m_{\tilde{f}}$. The 


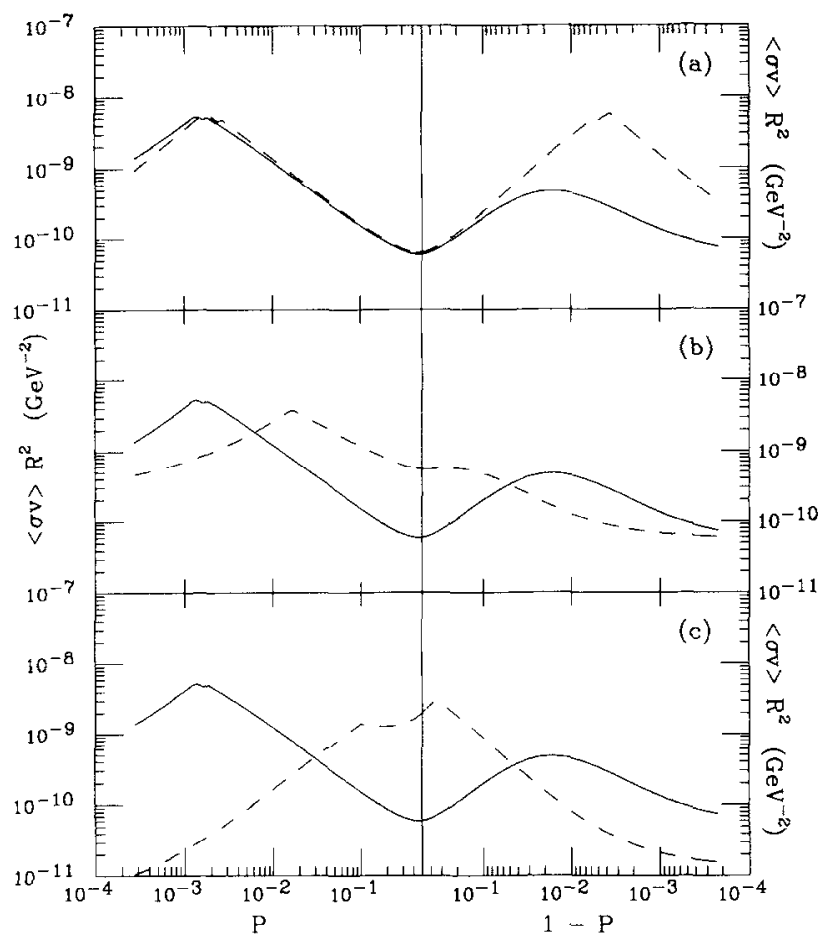

Fig. 3. $\langle\sigma v\rangle R^{2}$ as a function of the neutralino composition for $\tan \beta=8$ and for a fixed value for the neutralino mass $m_{X}=30 \mathrm{GeV}$. The solid curve refers to $m_{h}=50 \mathrm{GeV}, m_{\tilde{f}}=45 \mathrm{GeV}$. The dashed curves denote different choices for the mass parameters: (a) $m_{h}=50 \mathrm{GeV}$ and $m_{\tilde{f}}=150 \mathrm{GeV}$; (b) $m_{h}=120 \mathrm{GeV}$ and $m_{\tilde{f}}=45 \mathrm{GeV}$; (c) $m_{h}=120 \mathrm{GeV}$ and $m_{\tilde{f}}=150 \mathrm{GeV}$.

dashed lines correspond to the values: i) $m_{h}=50 \mathrm{GeV}$, $m_{\tilde{f}}=150 \mathrm{GeV}$ in Fig. 3a, ii) $m_{h}=120 \mathrm{GeV}, m_{\tilde{f}}=45$ $\mathrm{GeV}$ in Fig. 3 b, iii) $m_{h}=120 \mathrm{GeV}, m_{\tilde{f}}=150 \mathrm{GeV}$ in Fig. 3c. The solid line always denotes the case where $m_{h}=50 \mathrm{GeV}, m_{\tilde{f}}=45 \mathrm{GeV}$; this line, already shown in Fig. 2, is displayed here only for sake of comparison. In Fig. 3a we see that a marked difference between the two curves shows up at a mixed composition with gaugino dominance. This is expected since in this case sfermion-exchange diagrams are important; then, for the gaugino-dominated neutralinos, an increase of $m_{\tilde{f}}$ entails a sizeable suppression in the $\mathcal{X}-\mathcal{X}$ annihilation cross section with a subsequent enhancement of the relic abundance and then of the $R^{2}$ scaling factor. Fig. $3 \mathrm{~b}$ shows a similar effect of enhancement; however, here the increase in $R^{2}$, and then in $\langle\sigma v\rangle R^{2}$, occurs for the mixed compositions, since these are very sensitive to the value employed for $m_{h}$. Finally in Fig. $3 \mathrm{c}$ we notice that, when $m_{h}$ and $m_{\tilde{f}}$ are both large, the curve has a marked peak for $P \simeq 0.5$; as a matter of fact in this case rescaling is practically absent and $\langle\sigma v\rangle R^{2} \simeq$ $\langle\sigma v\rangle$.

In Fig. 4 the ratio of antiproton-to-proton integrated fluxes, $(\bar{p} / p)_{\text {int }}$, is displayed versus $P$ for $m_{\chi}=30$ $\mathrm{GeV}$ and $\tan \beta=2,8$. The $\bar{p}$ spectrum has been evaluated from Eq. (3) and the proton spectrum is taken from Ref. [26]: $\Phi_{p}\left(E_{p}\right)=1.93 v_{p}\left(E_{p} / \mathrm{GeV}\right)^{-2.7}$ $\mathrm{cm}^{-2} \mathrm{~s}^{-1} \mathrm{sr}^{-1} \mathrm{GeV}^{-1}\left(v_{p}\right.$ and $E_{p}$ are the proton velocity and energy). The modulation for both spectra is obtained with the procedure discussed above in Section 2.5. Both $p$ and $\bar{p}$ spectra have been integrated over the ranges: (a) $100 \mathrm{MeV} \leq T \leq 640 \mathrm{MeV}$; (b) $640 \mathrm{MeV} \leq T \leq 1580 \mathrm{MeV}$; and (c) $120 \mathrm{MeV} \leq$ $T \leq 860 \mathrm{MeV}$ in order to conform to the energy windows used in the experiments of Refs. [27,28]. The horizontal lines in Fig. 4 denote the corresponding upper bounds: $(\bar{p} / p)_{\text {int }}=2.8 \cdot 10^{-5}(85 \%$ C.L. $)$ for the interval a) $[27] ;(\bar{p} / p)_{\mathrm{int}}=6.1 \cdot 10^{-5}(85 \%$ C.L.) for the interval b) [27]; $(\bar{p} / p)_{\text {int }}=1.8 \cdot 10^{-5}$ ( $85 \%$ C.L.) for the interval c) [28]. It is remark- 


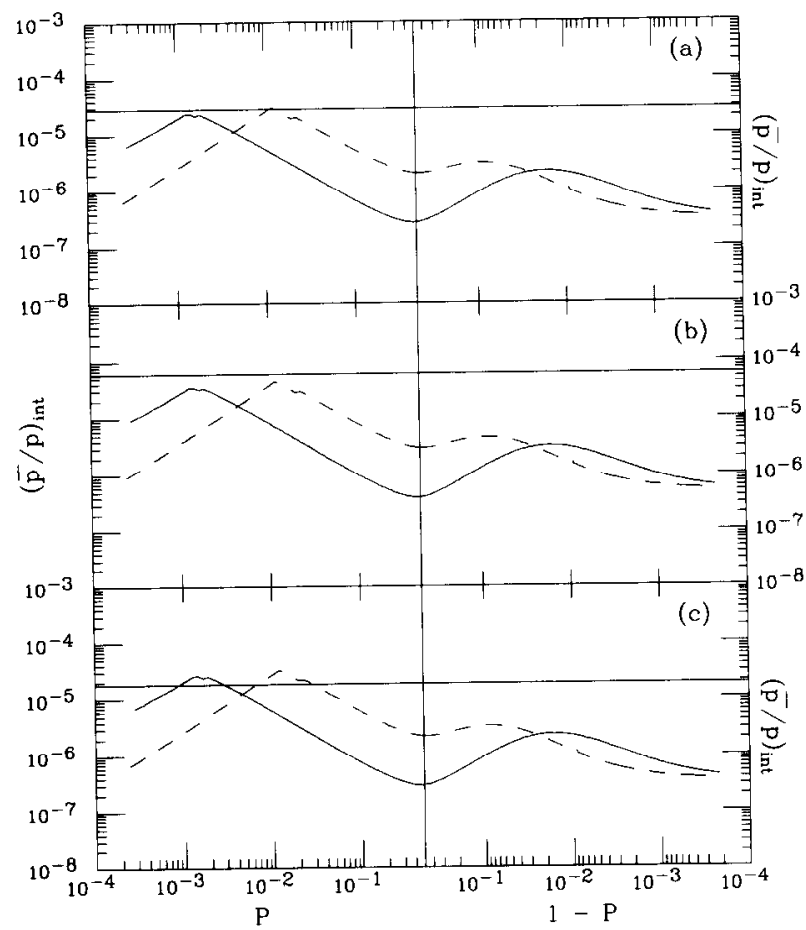

Fig. 4. Ratio of antiproton-to-proton integrated fluxes, $(\bar{p} / p)_{\text {int }}$, as a function of the neutralino composition for $m_{\chi}=30 \mathrm{GeV}$. The solid curve refers to $\tan \beta=8$, the dashed curve to $\tan \beta=2$. The mass parameters are: $m_{h}=50 \mathrm{GeV}$ and $m_{7}=45 \mathrm{GeV}$. The integration ranges are: (a) $100 \mathrm{MeV} \leq T \leq 640 \mathrm{MeV}$; (b) $640 \mathrm{MeV} \leq T \leq 1580 \mathrm{MeV}$; (c) $120 \mathrm{MeV} \leq T \leq 860 \mathrm{MeV}$. The horizontal lines are the corrisponding present upper bounds: (a) $(\bar{p} / p)_{\mathrm{int}}=2.8 \cdot 10^{-5}(85 \%$ C.L. $)[27]$; (b) $(\bar{p} / p)_{\mathrm{int}}=6.1 \cdot 10^{-5}$ (85\% C.L.) [27]; (c) $(\bar{p} / p)_{\text {int }}=1.8 \cdot 10^{-5}(85 \%$ C.L. $)[28]$.

able that the experimental sensitivities are already at the level of the maximum of the theoretical curves (at the representative points under discussion). This means that by improving these sensitivities one is able to start investigating the relic neutralinos by the measurement of $\bar{p} / p$.

Let us turn to the plots of the $\bar{p} / p$ ratio versus the $\bar{p}$ kinetic energy $T$. Fig. 5 shows how sensitively, at fixed $m_{\chi}$, this ratio depends on the neutralino composition. In this figure also the background due to the secondary antiprotons produced in cosmic-ray collisions in the interstellar medium is displayed [29].

The dependence on the neutralino mass of the plots of $\bar{p} / p$ versus $T$ is illustrated in Fig. 6 for three values of $P$ separately [30]. In Fig. $6 \mathrm{c}$ we notice that the ordering of the four curves for our signal is the usual one, expected on the basis of the dependence on $\mathrm{m}_{\chi}^{-2}$ in Eq. (3). However, in Figs. 6a, 6b this mass hierarchy turns out to be modified; this effect occurs when $2 m_{\chi}$ is close to one of the masses of the particles exchanged in the s-channel of the annihilation process. Then, in the present case, this happens for $m_{X}=30 \mathrm{GeV}$ which is close to the value used here for one of the neutral Higgs bosons.

It has to be pointed out that many uncertainties are involved in the theoretical predictions for the $\bar{p} / p$ signal, as it emerges from the derivation of the $\bar{p}$ flux presented above. Suffice it to mention the number of free parameters in MSSM and the wide range in the estimate of the confinement time $\tau_{\bar{p}}$.

Nevertheless, from the previous analysis it turns out that a substantial improvement in the measurement of the $\bar{p} / p$ ratio [31] can make this kind of investigation competitive in the future with other experimental strategies which aim at finding a sign of neutralino DM. This measurement shares with the measurements of other signals due to the $\chi-\chi$ annihilation in the halo $[6-10]$ the peculiar possibility of providing in- 


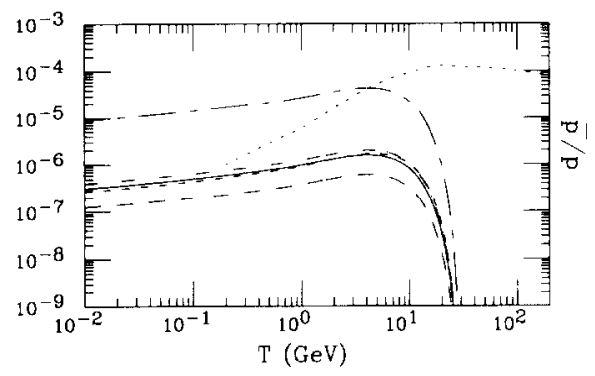

Fig. 5. Antiproton-to-proton flux ratio, $\bar{p} / p$, as a function of the kinetic energy $T$, for $m_{\chi}=30 \mathrm{GeV}$ and for different neutralino compositions: $P=10^{-3}$ (long-short dashed curve); $P=0.1$ (solid curve); $P=0.5$ (long dashed curve); $P=0.9$ (dash-dotted curve); $P=0.999$ (short dashed curve). The dotted curve is the background fiux of sccondary antiprotons, calculated in the leaky-box model [29]. Here $\tan \beta=8, m_{h}=50 \mathrm{GeV}$ and $m_{\tilde{f}}=45$ GeV.

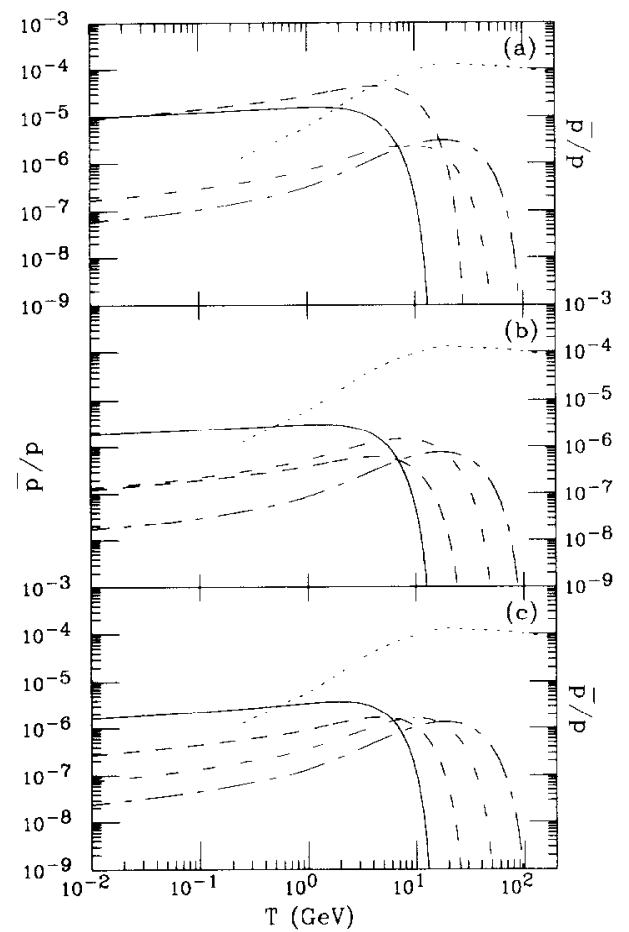

Fig. 6. Antiproton-to-proton flux ratio, $\bar{p} / p$, as a function of the kinctic energy $T$. Different curves refer to the following neutralino masses: $m_{X}=15 \mathrm{GeV}$ (solid curve); $m_{X}=30 \mathrm{GeV}$ (dashed curve); $m_{\chi}=60 \mathrm{GeV}$ (dash-dotted curve); $m_{X}=120 \mathrm{GeV}$ (long-short dashed curve). In (a) the neutralino composition is fixed at the value $P=10^{-3}$; in (b) $P=0.5$; in (c) $P=0.999$. The dotted curve is the background flux of secondary antiprotons, calculated in the leaky-box model [29]. Here $\tan \beta=8, m_{h}=50$ $\mathrm{GeV}$ and $m_{\tilde{f}}=1.2 m_{\chi}$, when $m_{\chi}>45 \mathrm{GeV}, m_{\tilde{f}}=45 \mathrm{GeV}$ otherwise.
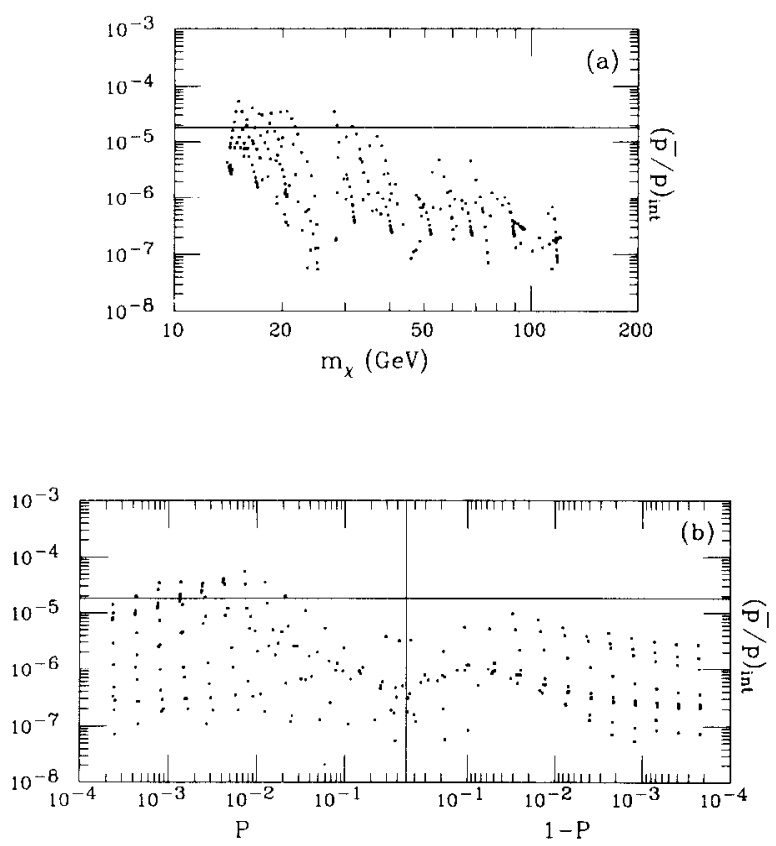

Fig. 7. Ratio of antiproton-to-proton integrated fluxes, $(\bar{p} / p)_{\mathrm{int}}$, as a function of the neutralino mass $m_{\chi}$ (a) and of the neutralino composition (b). The figure is presented in the form of a scatter plot obtained by varying the model parameters in the ranges: 10 $\mathrm{GeV} \leq M_{2} \leq 6 \mathrm{TeV}, 10 \mathrm{GeV} \leq|\mu| \leq 3 \mathrm{TeV}$. The integration interval is $120 \mathrm{MeV} \leq T \leq 860 \mathrm{MeV}$. The horizontal line is the corresponding upper bound $(\bar{p} / p)_{\text {int }}=1.8 \cdot 10^{-5}(85 \%$ C.L. $)$ [28]. Here $\tan \beta=8, m_{h}=50 \mathrm{GeV}$ and $m_{\tilde{f}}=1.2 m_{\chi}$. when $m_{\chi}>45 \mathrm{GeV}, m_{\tilde{f}}=45 \mathrm{GeV}$ otherwise.

formation about $\chi$-compositions with small mixings, which have little chances to be detected by other experimental means [32]. This point is illustrated in Fig. 7 where we give the $(\bar{p} / p)_{\text {int }}$ ratio versus $m_{X}(7 \mathrm{a})$ and versus $P(7 \mathrm{~b})$ in the form of a scatter plot, obtained by varying the two parameters $M_{2}$ and $\mu$ in the ranges $10 \mathrm{GeV} \leq M_{2} \leq 6 \mathrm{TeV}, 10 \mathrm{GeV} \leq|\mu| \leq 3$ $\mathrm{TeV}$, at fixed $\tan \beta=8$. These two plots show how the exploration may proceed inside the DM neutralino physical region, especially at small $P$ and small $m_{\chi}$, by increasing the experimental sensitivity in the measurement of $\bar{p} / p$.

The most serious problem for the detection of the neutralino signal under discussion is due to the uncertainty in the evaluation of the flux of secondary $\bar{p}$ 's produced by cosmic-ray collisions; this flux plays here the role of a background. Unfortunately, the calculation of this spectrum is affected by large uncer- 
tainties at small $\bar{p}$ kinetic energies, making the signalto-background discrimination difficult. Then a more accurate evaluation of this background is very much needed. However, it has to be emphasized that new stringent upper limits in the range of small energies would anyway provide useful constraints about the neutralino physical region.

\section{References}

[1] For an up-to-date review about experiments for direct detection of dark matter particles see L.Mosca, invited talk at the XIVth Moriond Workshop, 1994 (Proc., to appear).

12] A. Bottino, V. de Alfaro, N. Fornengo, G. Mignola and S. Scopel, Astroparticle Physics 2 (1994) 77, and references quoted therein.

[3] For a general overview about the experimental capabilities in this field see Proc. of the Sixth International Workshop on Neutrino Telescopes (Venezia 1994, Ed. Milla Baldo Ceolin), to appear.

[4] A. Bottino, N. Fornengo, G. Mignola and L. Moscoso: Signals of neutralino dark matter from Earth and Sun, University of Torino preprint DFTT $34 / 94$ (July 1994), and references quoted therein.

[5] Signals due to non-baryonic dark matter annihilations in the Large Magellanic Cloud have been considered in P. Gondolo, Nucl. Phys. (Proc. Suppl.) B 35 (1994) 148 (TAUP 93 Proceedings, Ed.s C. Arpesella, E. Bellotti and A. Bottino).

[6] L. Bergström and H. Snellman, Phys. Kev. D 37 (1988) 3737; S. Rudaz, Phys. Rev. D 39 (1989) 3549;

G.F. Giudice and K. Griest, Phys. Rev. D 40 (1989) 2549; A. Bouquet, P. Salati and J. Silk, Phys. Rev. D 40 (1989) 3168; L. Bergström, Phys. Lett. B 225 (1989) 372;

S. Rudaz and F.W. Stecker, Ap. J. 368 (1991) 406; V.S. Berezinsky, A. Bottino and V. de Alfaro, Phys. Lett. B 271 (1992) 122;

L. Bergström and J. Kaplan: Gamma ray lines from TeV dark matter, USITP-94-03 preprint.

[7] S. Rudaz and F.W. Stecker, Ap. J. 325 (1988) 16; F.W. Stecker and A.J.Tylka, Proc. 21 st ICRC (Adelaide, 1990 ), vol. 1, p.142.

[8] M. Urban, A. Bouquet, B. Degrange, P. Fleury, J. Kaplan, A.L. Melchior and E. Paré, Phys. Lett. B 293 (1992) 149.

[9] K. Freese and J. Silk, Phys. Rev. D 40 (1989) 3828; H.-U. Bengtsson, P. Salati and J. Silk, Nucl. Phys. B 346 (1990) 129.

[10] J.Silk and H. Bloemen, Ap. J. Lett. 313 (1987) L47; J.R. Ipser and P. Sikivie, Phys. Rev. D 35 (1987) 3695; V.S. Berezinsky, A.V. Gurevich and K.P. Zybin, Phys. Lett. B 294 (1992) 221;

J. Silk and A. Stebbins Ap. J. 411 (1993) 439;

V.S. Berezinsky, A. Bottino and G. Mignola, Phys. Lett. B 325 (1994) 136.
[11] F.W. Stecker, S. Rudaz and T.F. Walsh, Phys. Rev. Lett. 55 (1985) 2622.

[12] S. Rudaz and F.W. Stecker, Ap. J. 325 (1988) 16.

[13] J. Ellis, R.A. Flores, K. Freese. S. Ritz, D. Seckel and J. Silk Phys. Lett. B 214 (1988) 403.

[14] G. Jungman and M. Kamionkowski, Phys. Rev. D 49 (1994) 2316.

[15] J. Ellis, J.S. Hagelin, D.V. Nanopoutos, K. Olive and M. Srednicki, Nucl. Phys. B 238 (1984) 453.

[16] M. Drees, G. Jungman, M. Kamionkowski and M.M. Nojiri, Phys. Rev. D 49 (1994) 636.

[17] A. Bottino, V.de Alfaro, N. Fornengo, G. Mignola and $M$. Pignone, Astroparticle Physics 2 (1994) 67.

[18] T. Sjöstrand, Comp. Phys. Comm. 39 (1986) 347; Comp. Phys. Comm. 43 (1987) 367; CERN-TH 6488/92.

[19] The fragmentation functions for $c$ and $b$ quarks used here are the ones suggested by C.Peterson, D. Schletter, L. Schmitt and P.M Zerwas, Phys. Rev. D 27 (1983) 105.

[20] P. Mättig, Phys. Rep. 177 (1989) 141

[21] V.S. Berezinsky, S.V. Bulanov, V.A. Dogiel, V.L. Ginzburg and V.S. Ptuskin: Astrophysics of Cosmic Rays (NorthHolland, 1990) ch.3.

[22] J.N.Bahcall and R.M.Soneira, Ap. J. Suppl. 44 (1980) 73.

[23] J.A.R.Caldwell and J.P.Ostriker, Ap. J. 251 (1981) 61.

[24] L.A. Fisk, J. Geophys. Res. 76 (1971) 221.

[25] J.S. Perko, Astron. Astrophys. 184 (1987) 119.

[26] M.J. Ryan, J.F. Ormes, V.K. Balasubrahmanyan, Phys. Rev. Lett. 28 (1972) 985.

[27] S.W. Barwick et al., Proc. 21st ICRC (Adelaide, 1990), vol. 3, p.273.

[28] R.E. Streitmatter et al., Proc. 21st ICRC. (Adelaide, 1990), vol. 3, p.277.

[29] R.J. Protheroe, Ap. J. 251 ( 1981 ) 387.

[30] In order to explore a rather wide range in the neutralino mass, in Fig. 6 we have used as a representative $m_{\chi}$ value for a light neutralino the value $m_{\chi}=15 \mathrm{GeV}$, even if, strictly speaking, this is marginally excluded by the LEP data, which give $m_{\chi} \gtrsim 18 \mathrm{GeV}$ (at $90 \%$ C.L.), if GUT relations are applied (L. Roszkowski, Phys. Lett. B 252 (1990) 471; K. Hidaka, Phys. Rev. D 44 (1991) 927).

[31] The Balloon-borne Experiment with a Superconducting Solenoidal Spectrometer (BESS) is expected to reach for the $\bar{p} / p$ ratic the level of $10^{-6}$ (or even $10^{-7}$, by making use of the long duration ballooning capability) in an energy range: 120-600 MeV (K. Anraku, Proc.of the 23rd International Cosmic Rays Conference (Calgary 1993) p.156). A new project for a satellite experiment (PAMELA) is designed to reach the level of $0.5 \cdot 10^{-7}$ with the energy window: $100-500 \mathrm{MeV}$ (Dr. A. Morselli, private communication).

[32] This is due to the fact that in the case of the $\chi-\chi$ annihilation in the halo the signals depend quadratically on $\rho_{\chi}$ instead of linearly as in the cases of the direct detection and of the signals from the Earth and the Sun. 